\title{
Chawa Hildeschayn (1908-2007)la "doctoresse Jaffé”, directrice du Home de Profondsart
}

\author{
Catherine Massange
}

\section{(2) OpenEdition \\ 1 Journals}

Édition électronique

URL : http://journals.openedition.org/cmc/528

DOI : $10.4000 / \mathrm{cmc} .528$

ISSN : 2684-3080

\section{Éditeur}

Fondation de la Mémoire Contemporaine

\section{Édition imprimée}

Date de publication : 1 décembre 2011

Pagination : 393-404

ISSN : 1377-1256

\section{Référence électronique}

Catherine Massange, "Chawa Hildeschayn (1908-2007)la "doctoresse Jaffé", directrice du Home de Profondsart », Les Cahiers de la Mémoire Contemporaine [En ligne], 10 | 2011, mis en ligne le 01 décembre 2019, consulté le 16 novembre 2020. URL : http://journals.openedition.org/cmc/528 ; DOI : https://doi.org/10.4000/cmc.528 


\section{Chawa Hildeschayn (1908-2007) \\ la "doctoresse Jaffé", directrice du Home de Profondsart}

\section{Catherine Massange}

Dans l'immédiat après-guerre, ce fut l'Aide aux Israélites Victimes de la Guerre, (AIVG), qui se chargea des enfants dont les parents avaient été déportés'. L'AIVG entendait donner dans ses homes la meilleure éducation possible à ces enfants. Encore fallait-il disposer de moyens financiers très importants. En cela, l'aide de l'American Jewish Joint Distribution Committee, le Joint, fut fondamentale. Mais il était aussi indispensable de recruter un personnel adéquat. Il n'existait ni théorie éducative, ni diplôme particulier, ni formation professionnelle de nature à préparer les directeurs et directrices à faire face à leur tâche. Tous devaient improviser en fonction de leur sensibilité, de leur caractère, de leur passé. Les directeurs et directrices de ces homes jouissaient d'une grande autonomie et leur personnalité marqua profondément les enfants.

Le home de l'AIVG à Profondsart et sa directrice, le docteur Eva Jaffé (née Chawa Hildeschayn), étaient souvent cités en exemple. Les assistantes sociales du Ministère de la Reconstruction qui inspectaient les homes subventionnés exprimaient souvent leur admiration pour l'AIVG. L'une d'elles écrivait à propos de Profondsart : « Ce home est un véritable modèle au point de vue de l'éducation des enfants ${ }^{2}{ }^{2}$

\section{Émigrer}

Chawa Hildeschayn, ou la "doctoresse Jaffé" comme elle sera habituellement appelée ultérieurement, est née à Kozminek, près de Kalisz, dans la province de Łódź, en Pologne, le 14 octobre $1908^{3}$. Elle est la

\footnotetext{
${ }^{1}$ Sur l'histoire de l'AIVG, voir C. Massange, Bâtir le lendemain. L'Aide aux Israélites Victimes de la Guerre et le Service social juif de 1944 à nos jours, Bruxelles, 2002.

${ }^{2}$ Service public fédéral Sécurité sociale, Service des Victimes de la Guerre (SVG), Homes fermés. Enfants. Profondsart, « Visite d'inspection le 24 mai 1949 à Profondsart ».

${ }^{3}$ Fondation de la Mémoire contemporaine (FMC), fonds Jaffé, photocopie de la carte d'identité de Chawa Hildeschayn, 17 janvier 2000. Nous avons ici choisi la graphie « Hildeschayn », mais ellemême écrira son nom dans des documents officiels ou l'épellera de différentes manières:
} 
fille de Chaim Hildeschajm et de Liba Moszkowicz ${ }^{4}$. Elle est issue d'une famille nombreuse, très religieuse ${ }^{5}$.

Elle arrive en Belgique " pour étudier » en décembre 1929. Sa mère est alors déjà décédée 6 . Chawa est accompagnée de deux amies qui s'installent à Liège. L'une d'elles, Esther Zilberberg, communiste, s'engagera dans la Guerre d'Espagne ${ }^{7}$. De nombreuses raisons poussent ces jeunes Juives à quitter la Pologne pour venir étudier en Belgique ou ailleurs : le numerus clausus, l'antisémitisme, le manque de perspectives professionnelles et pour certaines, comme il semble que ce soit le cas pour Chawa Hildeschayn, un contexte familial religieux qu'elles jugent étouffant ${ }^{8}$. Entre 1918 et 1939, 524 étudiantes juives d'Europe de l'Est s'inscrivirent à l'ULB, dont la moitié environ à la Faculté des Sciences et de Médecine9.

Chawa vit à Bruxelles, où elle a trouvé un travail lui procurant un revenu indispensable pendant la durée de ses études ${ }^{10}$. Elle commence la médecine à l'Université libre de Bruxelles en 1930 et est diplômée en juillet 1937: elle est «docteur en médecine, chirurgie et accouchements » au grade scientifique et non au grade légal ${ }^{11}$. Elle ne peut donc pas travailler avec le statut d'indépendante et ouvrir un cabinet médical à son nom. Cette règlementation était censée favoriser le retour

Hildeschajm ou Hildesheim par exemple. Les enfants de Profondsart la connaissaient plutôt sous le nom de « doctoresse Jaffé » ou « Eva Jaffé ». On trouve aussi Kozminek ou Kozminck.

4 Archives générales du Royaume (AGR), Police des Étrangers (PE), dossiers individuels, 1.603.404.

${ }^{5}$ FMC, interview de Mina Buhbinder-Kostelaniec, 2011

${ }^{6}$ AGR, PE 1.603.404.

7 Interview de Jacob Jaffé et de Chawa Hildeschayn par Rudi Van Doorslaer, 1988 : l'auteur remercie Rudi Van Doorslaer de lui avoir communiqué ses notes et de lui avoir exposé ses souvenirs de cette rencontre. FMC, interview de Mina Buhbinder-Kostelaniec, 2011. Voir aussi le récit de vie d'Esther Zilberberg écrit par son fils : G. Waysand, Estoucha, Paris, 1997.

8 FMC, interview de Mina Buhbinder-Kostelaniec, 2011. L'auteur remercie Pascale FalekAlhadeff de son aide. Voir les travaux de Pascale Falek-Alhadeff dont la thèse s'intitule $A$ Precarious Life. East European Female Jewish Students in Interwar Belgium (Florence, 2011). Pascale Falek-Alhadeff a publié notamment : "Hélène Temerson (1896-1977) : parcours d'une universitaire juive d'Europe de l'Est », dans Les Cahiers de la Mémoire contemporaine, $\mathbf{n}^{\circ} 9$, Bruxelles, 2009-2010, pp. 135-167.

${ }^{9}$ P. Falek-Alhadeff, A Precarious Life..., op. cit., pp. 156-159.

${ }^{10}$ Interview de Jacob Jaffé et de Chawa Hildeschayn par Rudi Van Doorslaer, 1988. FMC, interview de Mina Buhbinder-Kostelaniec, 2011.

${ }^{11}$ Archives de l'Université libre de Bruxelles (AULB), fiche de Chawa Hildeschajm. FMC, fonds Jaffé, attestation de l'Université libre de Bruxelles, 1948. 
dans leur pays de jeunes diplômés venus suivre leurs études en Belgique $^{12}$.

Chawa Hildeschayn épouse Jacob Jaffé le 29 janvier 1938 à Ixelles ${ }^{13}$. Jacob Jaffé est né à Kalisz, en Pologne, le 3 décembre 1908. Kalisz comptait environ 89.000 habitants avant la Seconde Guerre mondiale, dont 20.000 Juifs $^{14}$. La mère de Jacob, Dinah Messing, y est également née. Jacob Jaffé arrive en Belgique en novembre 1929. Il vient suivre des études de chimie à l'Université libre de Bruxelles. Il dispose des papiers, passeport et visa nécessaires, et est donc autorisé à vivre en Belgique durant ses études ${ }^{15}$.

Jacob Jaffé est l'un des administrateurs de l'Association des Étudiants juifs de Bruxelles et l'un des représentants du groupe marxiste, Funk ("étincelle", en yiddish), une des composantes de cette association ${ }^{16}$. Chawa Hildeschayn est également active dans Funk. Elle accueillera par ailleurs temporairement et clandestinement son frère, Szlama, militant communiste qui avait été emprisonné en Pologne, avant qu'il ne parte combattre en Espagne. Elle-même récolte des fonds pour les républicains espagnols ${ }^{17}$. Le militantisme de Jacob et Eva Jaffé reste toutefois discret et n'attire pas les foudres de la police $^{18}$.

\section{L'Occupation}

En 1940, aux débuts de l'Occupation, Jacob Jaffé travaille comme assistant en chimie à l'Université libre de Bruxelles. Chawa est engagée comme assistante du docteur bruxellois Delchef ${ }^{19}$.

12 P. Falek-Alhadeff, A Precarious Life..., op. cit., pp. 112-113. Fr. Caestecker et J. De Bock, « Historiek van de studentenmigratie naar België 1830-1994 », dans Fr. Caestecker et A. Rea Migreren voor een diploma. Studenten van buiten de Europese Unie in het Belgisch hoger onderwijs, Leuven, 2009, pp. 28-29.

${ }^{13}$ AGR, PE 1.603.404, extrait du registre aux actes de mariage de la Commune d'Ixelles.

14 http://www.ushmm.org/wlc/en ; United States Holocaust Memorial Museum, Holocaust Encyclopedia (novembre 2011).

15 AGR, PE 1.603.404.

${ }^{16}$ R.Van Doorslaer, Enfants du ghetto. Juifs révolutionnaires de Belgique (1925-1940), Bruxelles, 1997, p. 91.

${ }^{17}$ Interview de Jacob Jaffé et de Chawa Hildeschayn par Rudi Van Doorslaer, 1988.

18 AGR, PE 1.603.404.

${ }^{19}$ FMC, fonds Jaffé, Curriculum vitae de Chawa Hildeschayn. 
En décembre 1940, Jacob Jaffé et son épouse s'inscrivent au Registre des Juifs ${ }^{20}$. Mais Chawa se méfie immédiatement de l'Association des Juifs en Belgique, créée par l'occupant ${ }^{21}$. Ils ne s'y enregistrent pas alors que cette démarche était obligatoire ${ }^{22}$.

Jacob Jaffé sera obligé d'abandonner son poste d'assistant à l'Université libre de Bruxelles ${ }^{23}$. Étant médecin, Chawa aurait pu trouver un emploi dans une des institutions de soins mises en place par les autorités occupantes qui, appliquant leur politique de ségrégation, prévoyaient que les Juifs soient soignés uniquement par des Juifs dans des établissements, tels que l'Hôpital Israélite ou le home pour Juifs âgés de Scheut (Anderlecht, Bruxelles), contrôlés par elles ou leurs intermédiaires ${ }^{24}$.

La clandestinité s'impose. En 2000, Eva Jaffé entreprendra des démarches pour que les personnes qui l'ont cachée pendant l'Occupation, Maurice et Élise Goisse ainsi que leur fille Nelly, soient reconnues comme Justes parmi les Nations par l'État d'Israël. Eva Jaffé expliquera : « Je venais d'avoir une petite fille, Aline, le 4 avril 1942. La situation commençait à devenir dangereuse. Un collègue, le docteur Bailleux qui travaillait avec moi dans le service du docteur Delchef m'a indiqué une petite pension de famille située à Céroux-Mousty. N'ayant pas d'argent, je n'ai pu rester plus d'un mois dans cette pension. Je me suis présentée avec ma petite fille âgée de 4 à 5 mois à la ferme la plus proche. J'ai exposé mon problème, j'ai expliqué les risques qu'ils pouvaient encourir, mais sans hésiter ils ont accepté de m'héberger avec ma petite fille. $»^{25}$

Jacob Jaffé lui, continue à travailler: il est employé comme chimiste dans le laboratoire Alliance Européenne SA, rue Gallait à

\footnotetext{
${ }^{20}$ MJB, Registre des Juifs.

21 Sur l'AJB, voir J.-Ph. Schreiber - R. Van Doorslaer (éds.), Les curateurs du ghetto. L'Association des Juifs en Belgique sous l'occupation nazie, Bruxelles, 2004.

${ }^{22}$ L'auteur remercie Laurence Schram (Kazerne Dossin) pour les renseignements communiqués.

${ }^{23}$ SVG, dossier Statut de Jacob Jaffé.

24 FMC, fonds Jaffé, attestation de Chaïm Perelman, 16 juillet 1948. FMC, fonds Jaffé, Curriculum vitae de Chawa Hildeschayn. À propos des médecins de l'Hôpital Israélite et de Scheut, voir : C. Massange, « L'Hôpital israélite de Bruxelles (1943-1944) », dans Les Cahiers de la Mémoire Contemporaine, ${ }^{\circ}$ 7, 2006-2007, pp. 13-36 et C. Massange - Th. Delplancq, « L'Hospice de Scheut (1943-1944) », dans Les Cahiers de la Mémoire Contemporaine, nº 5, 2003-2004, pp. 1334 .

${ }^{25}$ FMC, fonds Jaffé, témoignage de Chawa Hildeschayn-Jaffé, fax pour l'Association belge « L'Enfant Caché », 2000.
} 
Schaerbeek ${ }^{26}$. C'est là que la Gestapo l'arrête le 25 décembre 1943. Jacob Jaffé était persuadé qu'il avait été dénoncé. Un ingénieurchimiste qui travaillait avec lui expliquera en 1952: «J'ignore les motifs de l'arrestation de Jaffé. Il y a tout lieu de croire qu'elle a fait suite à une dénonciation car les boches qui sont venus l'arrêter un soir à l'usine étaient munis de sa photographie et s'étaient rendus directement par leurs seuls moyens au laboratoire où travaillait Jaffé et qui était situé au quatrième étage d'un bâtiment sis au fond d'une cour profonde. " $^{27}$ Des voisins attesteront en décembre 1944 « avoir vu emporter par la Gestapo, le 30 décembre 1943, tout ce que se trouvait dans l'appartement » des Jafféers.

Jacob Jaffé sera d'abord emmené dans les locaux de la Gestapo à l'avenue Louise. Il ne révèlera rien de l'endroit où se cache sa famille. Il est transféré à la caserne Dossin à Malines et déporté à Auschwitz par le XXIII e convoi du 15 janvier 1944. Sa sœur, Hawa, qui l'avait suivi en Belgique, ainsi que l'époux de Hawa, Morduch Kostelaniec, sont également déportés : elle, par le transport XXIIA le 20 septembre 1943, lui le 19 avril 1943, par le XX $\mathrm{XX}^{\mathrm{e}}$ convoi ${ }^{29}$. À Auschwitz, Jacob Jaffé sera mis au travail comme chimiste ${ }^{30}$.

Eva Jaffé accouchera donc de leur fils Marc, le 21 janvier 1944, alors que Jacob Jaffé vient d'être déporté.

\section{Profondsart}

À la Libération, Eva Jaffé est seule avec ses deux enfants. Elle attend son mari, dont elle n'a aucune nouvelle. Elle racontera que c'est Fela Perelman, qu'elle connaissait bien, qui lui propose alors de devenir directrice de home ${ }^{31}$. Fela Perelman-Liwer, l'épouse de Chaïm Perelman, s'occupa des homes, durant les premiers temps de l'AIVG, notamment en cherchant des bâtiments adéquats. Ni elle, ni son mari,

\footnotetext{
${ }^{26}$ Kazerne Dossin, « Enveloppe Reliques », Jaffé (Jacob), certificat de travail de Jacob Jaffé, 5 novembre 1942.

${ }^{27}$ SVG, dossier Statut de Jacob Jaffé.

${ }^{28}$ Ibid.

${ }^{29}$ Mecheln-Auschwitz 1942-1944: La destruction des Juifs et des Tsiganes de Belgique. 28 transports, 18.522 portraits, Bruxelles, 2009, t. 4, p. 178, p. 215, t. 3 (photos), p. 288, p. 253, p. 160.

${ }^{30}$ Interview de Jacob Jaffé et de Chawa Hildeschayn par Rudi Van Doorslaer, 1988. FMC, interview de Mina Buhbinder-Kostelaniec, 2011.
} 
figure historique du Comité de Défense des Juifs, l'organisation de Résistance qui aida des milliers de Juifs à se cacher durant l'Occupation, n'assumèrent durablement des responsabilités au sein de l'AIVG ${ }^{32}$. Fela Perelman ne semble pas croire à la possibilité d'un retour de Jacob Jaffé. Eva ne perd pas l'espoir ${ }^{33}$. Et Jacob, après avoir été transféré d'Auschwitz à Buchenwald, sera de fait libéré le 11 avril 1945 et rentrera en Belgique en mai $1945^{34}$.

Le home de Profondsart est un château, situé dans le Brabant wallon. Contrairement aux autres homes de l'AIVG, il ne connaîtra qu'une directrice, Eva Jaffé. L'Euvre nationale de l'Enfance encourage sa candidature $^{35}$. Les pensionnaires des autres établissements de l'AIVG voyaient directeurs, directrices et personnel éducatif se succéder régulièrement, alors que l'AIVG aurait voulu assurer aux enfants continuité et stabilité affectives. Le personnel de Profondsart compte par ailleurs une dizaine de membres ${ }^{36}$.

En 1945, l'AIVG prévoit dans ses homes une trentaine de places pour les tout-petits de moins de 3 ans, une quarantaine pour les petits de 3 à 6 ans, 170 places environ pour les plus de 14 ans, et près de 300 places pour les 6-14 ans. Les enfants dans cette tranche d'âge sont donc les plus nombreux ${ }^{37}$. Et Profondsart, ouvert en juillet 1945, leur est destiné. Le nombre maximum de pensionnaires est estimé à 55 . En 1947, le home abrite 47 enfants, 19 filles et 28 garçons, de 8 à 16 ans ; en 1948, 35 ; en octobre 1949, 25. Le home ferme à l'été $1950^{38}$.

L'AIVG était particulièrement fière du home de Profondsart : un rapport de 1948 souligne les excellents résultats scolaires des enfants du home ${ }^{39}$. Irène Rosenstein-Zmigrod, assistante sociale de l'AIVG, trouve que : « $\grave{A}$ tous points de vue, ce fut vraiment une des plus belles

${ }^{31}$ FMC, interview d'Eva Jaffé par Vincent Decaestecker, 1998.

32 J.-Ph. Schreiber (éd.), Dictionnaire biographique des Juifs de Belgique. Figures du judaüsme belge $X I X^{e}-X X^{e}$ siècles, Bruxelles, 2002, pp. 271-275.

33 FMC, interview d'Eva Jaffé par Vincent Decaestecker, 1998.

${ }^{34}$ SVG, dossier Statut de Jacob Jaffé.

${ }^{35}$ SVG, documents De Lathouwer, classeur 1320, pp. 247-250.

${ }^{36}$ SVG, Homes fermés. Enfants. Profondsart, « Rapport d'inspection du 17.11.1949 ».

${ }^{37}$ SSJ, « Aide aux Israélites Victimes de la Guerre. Section Enfance. Rapport d'activité », 31 décembre 1945.

${ }^{38} \mathrm{SVG}$, Homes fermés. Enfants.

${ }^{39} \mathrm{SSJ}$, « Aide aux Israélites Victimes de la Guerre asbl. Rapport d'activité pour l'année 1948 ». 
maisons que nous ayons eues $\gg .^{40}$ Eva Jaffé accordait une énorme importance à l'instruction et à la culture et s'efforçait de communiquer aux enfants l'amour de la lecture, de la musique ${ }^{41}$. L'inspectrice du Ministère de la Reconstruction est admirative : "On chercherait en vain des griefs à faire à la marche de ce home $\$^{42}$ Elle souligne la responsabilité et les mérites de la directrice : « Elle connaît chaque enfant et possède sa confiance. Elle peut donc plus facilement le conseiller et le diriger dans les études, le choix d'un métier ou lors d'une décision à prendre. Il en est de même au point de vue santé ; étant médecin, elle connaît l'état médical de chacun et le suit de façon attentive. Aussi la majorité des enfants de Profondsart remporte-t-elle de grands succès scolaires, car chaque enfant fait les études qui sont appropriées à ses possibilités. De plus, il reçoit au home des leçons supplémentaires, si cela lui est utile. $»^{43}$ Elle ajoute : «Il est incontestable que la direction du home a une influence primordiale $\$ .{ }^{44}$ Ce qu'elle apprécie aussi particulièrement est que «la discipline nécessaire dans toute maison d'enfants y règne mais sans contrainte aucune $»^{45}$. Cet équilibre particulièrement difficile à maintenir dépend pour beaucoup de l'habileté de la directrice.

L'inspectrice du Ministère apprécie la qualité de l'hygiène et de la nourriture du home, en soulignant à nouveau que la directrice est médecin. On ne mange pas casher à Profondsart. Eva Jaffé revendique personnellement sa laïcité. Elle essayera de communiquer aux enfants une culture juive chaleureuse, illustrée par des fêtes et des chants ${ }^{46}$. Beaucoup lui en seront reconnaissants.

Les enfants de Profondsart rédigent un journal : Échos. Dans le numéro 2 de juin 1947, une jeune fille de 15 ans écrit : «La doctoresse a promis une nouvelle lecture aux filles! Quelle joie! " « Nous savons que ce sera quelque chose de bien, qui forcément captivera notre attention. » L'article évoque d'autres lectures et se termine par les mots :

\footnotetext{
${ }^{40}$ Interview d'Irène Rosenstein-Zmigrod par Vincent Decaestecker, 1998.

${ }^{41}$ FMC, interview de Mina Buhbinder-Kostelaniec, 2011.

42 SVG, Homes fermés. Enfants. Profondsart, « Rapport d'inspection du 7 octobre 1949 ».

${ }^{43}$ SVG, Homes fermés. Enfants. Profondsart, « Rapport d'inspection du 17.11.1949 ».

${ }^{44}$ SVG, Homes fermés. Enfants. Profondsart, « Visite d'inspection le 24 mai 1949 à Profondsart ».

45 SVG, Homes fermés. Enfants. Profondsart, «Visite d'inspection faite le 7 avril 1949 à Profondsart ».

${ }^{46}$ FMC, interview d'Eva Jaffé par Vincent Decaestecker, 1998.
} 
« Nous bâtirons un monde nouveau de nos propres efforts, nous gagnerons le pain dont nous aurons besoin, pour nous et les nôtres, nous n'aurons besoin d'aucune charité, nous serons libres !!! »77

Une des pensionnaires de Profondsart s'en souvient comme d'un lieu merveilleux et d'une période d' « harmonie », de "douceur », d'un « sentiment de liberté ${ }^{48}$. Profondsart dispose d'un bassin de natation. Les enfants attendent avec impatience les beaux jours, le remplissage du bassin et les premiers plongeons de l'été : «Jamais ces heureux moments ne s'effaceront de notre mémoire. ${ }^{49}$

Eva et Jacob Jaffé vivent donc avec leurs enfants dans une dépendance du château ${ }^{50}$. Mina Kostelaniec se souvient que son oncle, Jacob Jaffé, qui n'avait officiellement aucune fonction dans le home, s'occupait des enfants en animant des ateliers de « reliure, modelage, sculpture, marqueterie, tapisserie, modélisme, photographie $»^{51}$. Ces activités sont appréciées par l'inspectrice du Ministère : «L'enfant qui ne construit pas détruit. On a très bien compris cela à Profondsart. $»^{52}$

Bien sûr, il n'existait pas d'institution susceptible de répondre parfaitement aux traumatismes que venaient de subir les enfants : parlant de Profondsart, certains se souviennent aussi de la solitude dont ils souffraient malgré la convivialité du groupe, du poids du silence malgré les jeux et les rires, d'une maladresse ou d'une peur face à la sexualité malgré les possibilités de dialogue et de communication et la volonté de donner un minimum d'éducation sexuelle aux enfants ${ }^{53}$.

Les homes de l'AIVG disposent d'une grande autonomie. Par contre, ce qui relève de la responsabilité directe du conseil d'administration de l'AIVG, ce sont l'ouverture et la fermeture de ces homes, qui dépendent des impératifs budgétaires. Profondsart est fermé pendant

${ }^{47}$ FMC, fonds Jaffé, texte « Lecture au dortoir » signé « Paulette (15 ans) », dans Échos ${ }^{\circ}{ }^{2}$, juin 1947, p. 4.

${ }^{48}$ FMC, interview de Liliane Levit, 1999. Voir aussi l'interview d'Adolphe Nysenholc par Vincent Decaestecker, 1998.

49 FMC, fonds Jaffé, texte « Notre bassin de natation » signé « Minna (12 ans) » et « Hélène (13 ans) », dans Échos n ${ }^{\circ} 2$, juin 1947, pp. 5-6.

${ }^{50} \mathrm{FMC}$, interview de Mina Buhbinder-Kostelaniec, 2011.

${ }^{51}$ Le Livre des homes. Enfants de la Shoah. AIVG 1945-1959, sous la direction d'Adolphe Nysenholc, Bruxelles, 2004, p. 47.

52 SVG, Homes fermés. Enfants. Profondsart, «Visite d'inspection du 16 décembre 1947 ». Archives du Service social Juif (SSJ), procès-verbal du conseil d'administration de l'AIVG du 28 août 1950.

${ }^{53}$ FMC, interview de Liliane Levit, 1999. FMC, interview de Mina Buhbinder-Kostelaniec, 2011. 
l'été 1950. L'AIVG essaie dans la mesure du possible de déplacer les enfants durant les vacances d'été de façon à ne pas perturber leur scolarité, un changement de home entraînant l'inscription dans un nouvel établissement scolaire ${ }^{54}$.

Le 28 août 1950, le conseil d'administration de l'AIVG « remercie chaleureusement Mme Jaffé qui, pendant cinq ans, c'est-à-dire du jour de l'ouverture du Home jusqu'à sa fermeture a dirigé celui-ci avec une compétence et un dévouement exceptionnels. [...] L'AIVG est obligée avec un vif regret de fermer le Home de Profondsart qui était incontestablement un de ses meilleurs. ${ }^{55}$

\section{Après Profondsart}

En septembre 1950, la famille Jaffé doit donc quitter Profondsart. Jacob Jaffé sollicite sa naturalisation et devient belge en $1951^{56}$. Il poursuit sa carrière à l'Université libre de Bruxelles ${ }^{57}$. Il demandera sa reconnaissance comme prisonnier politique; comme beaucoup d'autres Juifs, il n'obtiendra finalement pas le titre de prisonnier politique, mais aura droit au bénéfice du statut ${ }^{58}$. Jacob et Eva se diront en rapport avec la Résistance pendant l'Occupation (lui, pour la presse clandestine; elle, pour l'aide aux enfants cachés), sans revendiquer d'appartenance à un mouvement précis ${ }^{59}$.

Eva Jaffé ouvrit un cabinet médical privé après avoir repris des études : elle suivit des cours à l'Université à Bologne, en Italie, et fit homologuer son diplôme ${ }^{60}$.

Elle vécut jusqu'à l'âge de 98 ans. Elle eut la douleur de perdre son fils dans les années 70, puis son mari ainsi que sa fille, décédés en 1997. Mais elle connut ses petits-enfants et arrière-petits-enfants. Elle mou-

\footnotetext{
54 SSJ, « Rapport à l'Assemblée Générale de l'AIVG du 5 mars 1951 ». SSJ, procès-verbal du conseil d'administration de l'AIVG du 28 août 1950

${ }^{55} \mathrm{SSJ}$, procès-verbal du conseil d'administration de l'AIVG du 28 août 1950.

${ }^{56}$ AGR, dossier Indigénat de Jacob Jaffé.

${ }^{57}$ AULB, fiche de Jacob Jaffé.

${ }^{58}$ SVG, dossier Statut de Jacob Jaffé.

${ }^{59}$ SVG, dossier Statut de Jacob Jaffé. FMC, fonds Jaffé, Curriculum vitae d'Eva Jaffé. FMC, attestation de Chaïm Perelman, 16 juillet 1948.

${ }^{60}$ FMC, interview de Mina Buhbinder-Kostelaniec, 2011.
} 
rut le 12 janvier 2007. Ses cendres furent dispersées au cimetière de Saint-Gilles à Uccle ${ }^{61}$.

En 2002, Eva Jaffé avait transmis un message aux anciens pensionnaires de Profondsart: "Je souhaite à tous mes anciens enfants, aujourd'hui parents, grands-parents, joie, santé, amour, amitié et solidarité. Et je les remercie pour le bonheur que j'ai eu de vivre cette riche et unique expérience $\${ }^{62}$

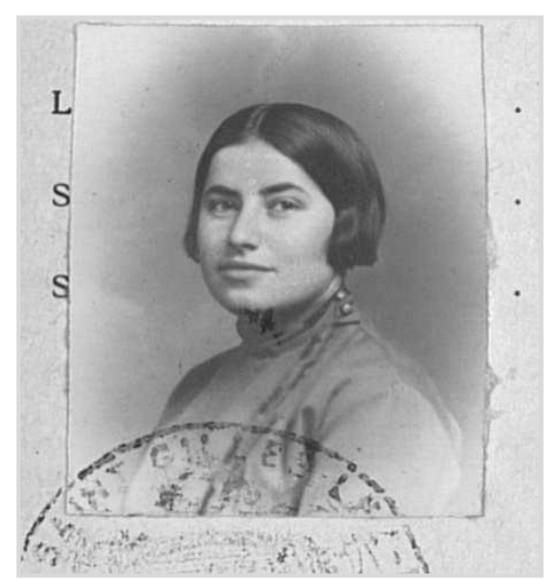

Chawa Hildeschayn vers 1929.

(C) AGR, PE, photo Kazerne Dossin.

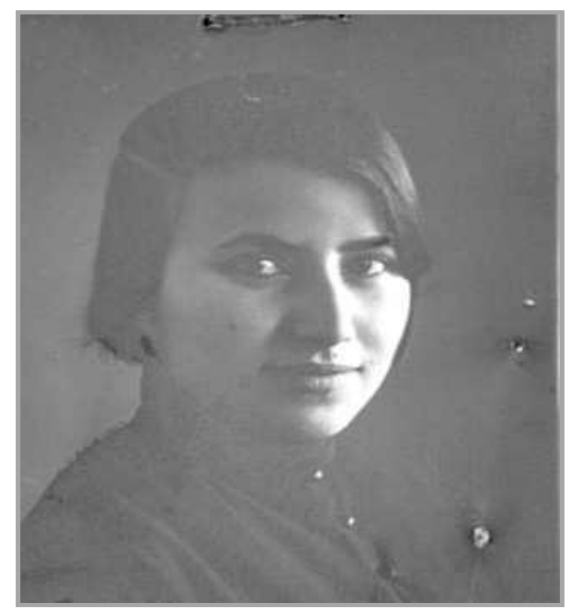

${ }^{61}$ Faire-part de décès de Chawa Hildeschayn (www.enaos.net).

${ }^{62}$ Le Livre des homes... op. cit., p. 67. 


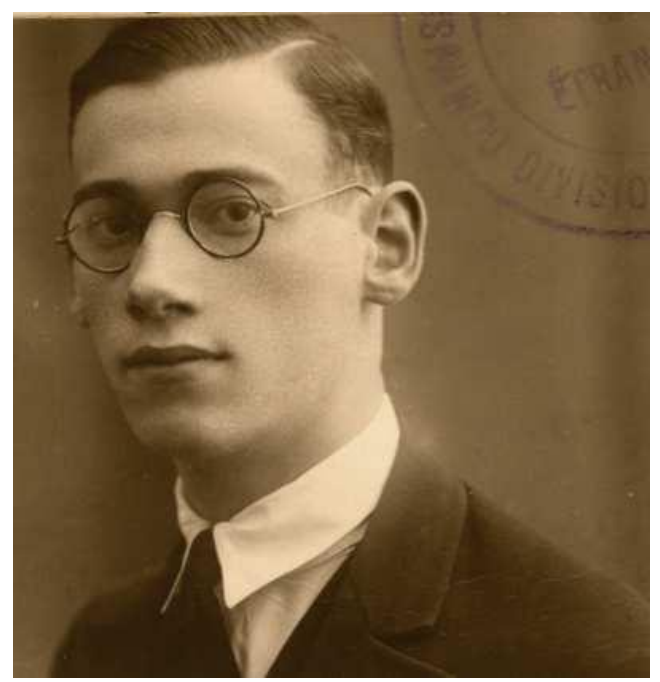

Jacob Jaffé vers 1929.

(C) $\mathrm{GGR}, \mathrm{PE}$, photo Kazerne

Dossin.

Eva et Jacob Jaffé au home de Profondsart.

(Souvenirs d'Enfance.

Homes de l'AIVG,

1944-1958).

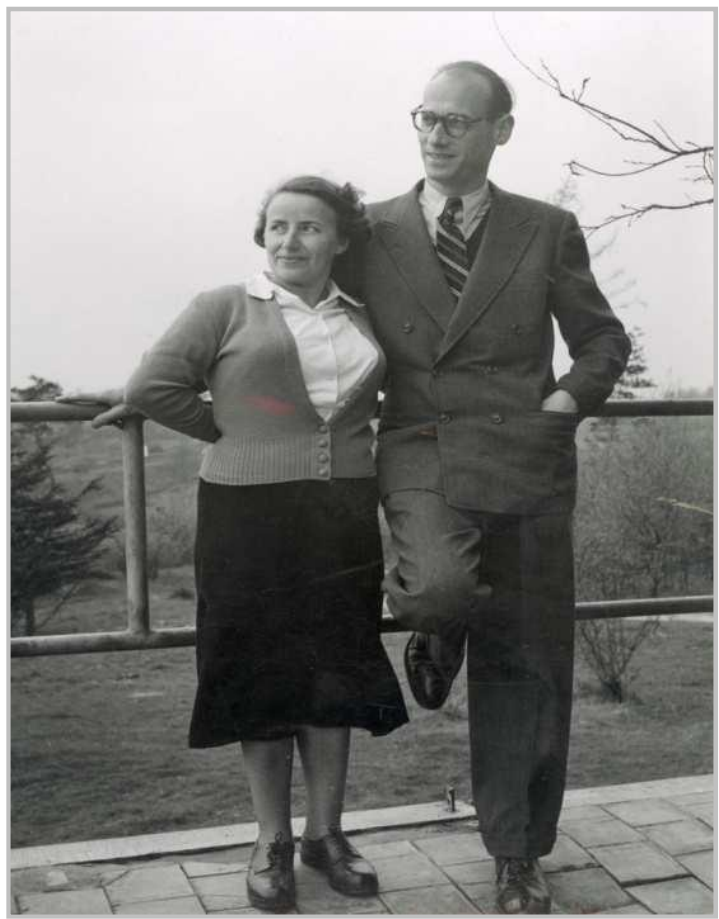

403 


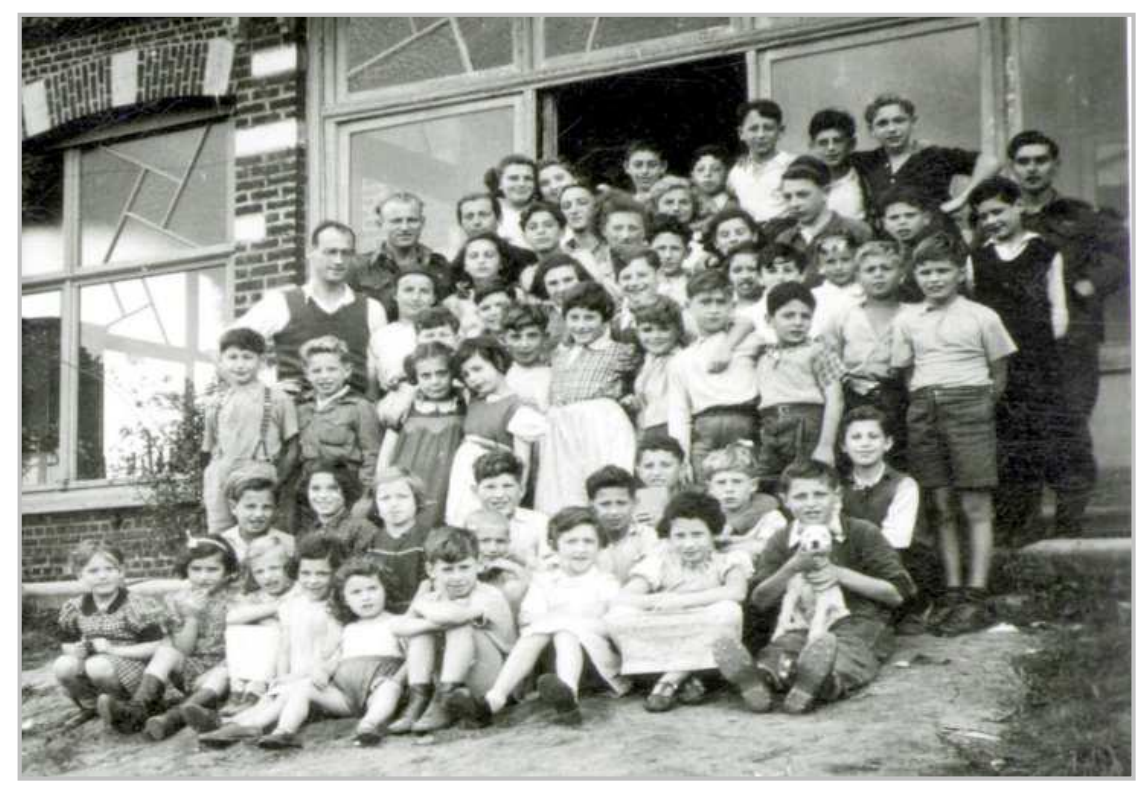

Le home de Profondsart (Souvenirs d'Enfance. Homes de l'AIVG, 1944-1958).

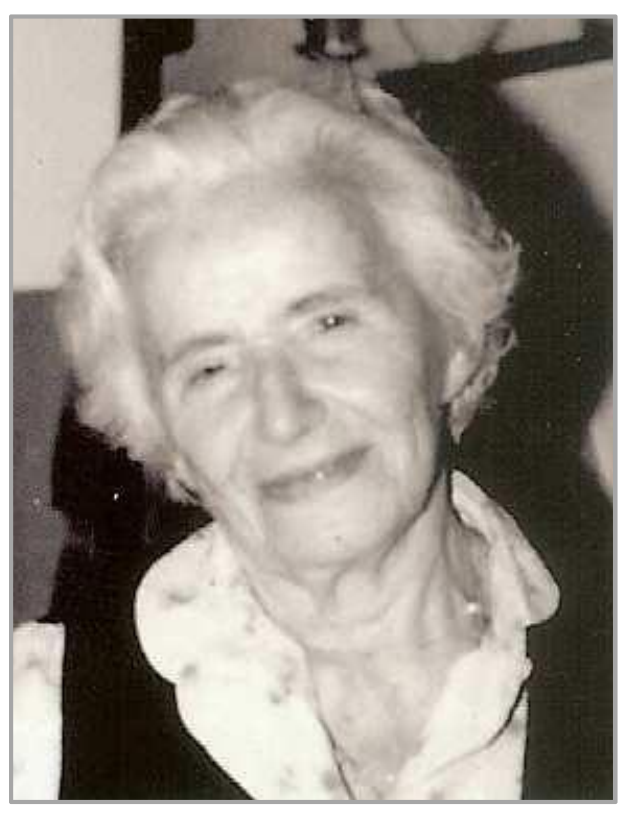

Chawa Jaffé-Hildeschayn. (C) UPJB. 